\title{
LA FAMILIA ANTE LA PANDEMIA POR LA COVID-19
}

THE FAMILY IN THE FACE OF THE COVID-19 PANDEMIC

José L. Huerta-González*

División de Consulta Externa, Hospital Central Dr. Ignacio Morones Prieto, San Luis Potosí, S.L.P., México

RESUMEN: La pandemia por la enfermedad por coronavirus 2019 (COVID-19) ha generado múltiples complicaciones psicosociales y económicas familiares en México, tales como disminución de actividades laborales, pérdida de empleos, cierre de empresas y pobreza súbita; los efectos familiares estructurales y funcionales incluyen incertidumbre, violencia, adicciones y trastornos psicológicos. El confinamiento doméstico como medida preventiva ha ocasionado frustración, enojo, disfunción familiar y estrés. Los fallecimientos la ubican como causa importante de mortalidad; además de las implicaciones del duelo, la familia enfrenta conflictos intrafamiliares, así como dificultades económicas, domésticas y laborales. Se ha incrementado el hacinamiento y la promiscuidad habitacional y ha afectado más a familias nucleares, extensas y seminucleares. Se recomiendan intervenciones específicas ante los problemas psicosociales y económicos, así como el abordaje integral que incluya la atención oportuna a personas contagiadas, el trabajo familiar conjunto, la evaluación del tipo, ciclo vital y funcionalidad familiar y el nivel socioeconómico, escolaridad y ocupación de los padres. Se deben investigar también las características de las viviendas, número de integrantes, enfermedades crónicas, apego terapéutico, disponibilidad de servicios médicos y acceso a redes sociales de apoyo. La pandemia ha representado un reto familiar por la adaptación para enfrentar situaciones desconocidas y complejas.

Palabras clave: Cuarentena. Infecciones por coronavirus. Relaciones familiares.
ABSTRACT: The coronavirus disease 2019 (COVID-19) pandemic has generated multiple family psychosocial and economic complications in Mexico, such as a decrease in work activities, loss of jobs, business closings and sudden poverty; structural and functional family effects include uncertainty, violence, addictions and psychological disorders. Domestic confinement as a preventive measure has caused frustration, anger, family dysfunction and stress. Deaths rank it as an important cause of mortality; in addition to the implications of grief, the family faces intra-family conflicts, as well as economic, domestic and work difficulties. Overcrowding and housing promiscuity have increased, affecting more nuclear, extended and semi-nuclear families. Specific interventions are recommended for psychosocial and economic problems, as well as a comprehensive approach that includes timely care for infected people, joint family work, evaluation of the type, life cycle and family functionality, and the socioeconomic level, education and occupation of the parents. The characteristics of the dwellings, number of members, chronic diseases, therapeutic attachment, availability of medical services and access to support social networks should also be investigated. The pandemic has represented a family challenge for adaptation to face unknown and complex situations.

Key words: Quarantine. Coronavirus infections. Family relationships.
Correspondencia:

*José L. Huerta-González

E-mail: jose_luishuerta@ hotmail.com
Fecha de reepción: 23-09-2020

Fecha de aceptación: 11-10-2020
Disponible en internet: 11-11-2020

Rev Mex Med Fam. 2020;7:109-14

DOI: 10.24875/RMF.20000130

2007-9710 / (C) 2020 Federación Mexicana de Especialistas y Residentes en Medicina Familiar. Publicado por Permanyer. Este es un artículo open access bajo la licencia CC BY-NC-ND (http://creativecommons.org/licenses/by-nc-nd/4.0/). 


\section{INTRODUCCIÓN}

La pandemia por la enfermedad por coronavirus 2019 (COVID-19) es un evento crítico paranormativo, repentino, caracterizado por pérdidas económicas, de contacto físico, certidumbre, autonomía y movilización. En general, ha afectado en mayor o menor medida a toda la sociedad, en función de múltiples variables y factores de riesgo.

Al día de hoy, esta pandemia es una de las primeras causas de mortalidad en México y muestra importantes características psicológicas, económicas y sociales que la diferencian de otros problemas de salud, tales como la disminución de las actividades laborales, la pérdida masiva de empleos y el cierre de fuentes de trabajo, situaciones que han impactado directamente en las condiciones de la vida familiar. Aparte del riesgo de contagio, los miembros de una familia con una persona infectada por la COVID-19 se preocupan por los cuidados que el paciente requiere, su evolución y pronóstico. Los problemas, sobre todo los económicos, se incrementan cuando hay más de una persona contagiada debido a los gastos por su atención y a los que surgen al presentarse un deceso ${ }^{1}$. Si se trata del padre, la madre o quien funja como proveedor o jefe de familia, los efectos pueden ser más graves. Las familias sin contagios ni defunciones presentan las mismas consecuencias, excepto por el proceso de duelo, que se asocian generalmente a la pérdida de su fuente de ingresos, a la incertidumbre y al confinamiento doméstico.

La incertidumbre se presenta tanto en las familias afectadas directamente como en las que solo han tenido otro tipo de repercusiones. Se caracteriza por la inquietud secundaria a la inseguridad, desconfianza y falta de certeza sobre las implicaciones y consecuencias de la pandemia. Manifiesta el grado de duda y desconocimiento acerca de este fenómeno, lo cual, aunado al bajo nivel de escolaridad, desinformación y pobre nivel económico, da lugar a incredulidad e indiferencia por un lado y a temor por el otro, situaciones que dificultan la práctica de las medidas preventivas implementadas, entre otros efectos. Quizá porque la mayor parte de las familias no han comprendido el fenómeno ni sus consecuencias, se generan expectativas poco realistas y se buscan culpables y explicaciones por doquier, como una forma de mantener la esperanza y de lidiar con la angustia causada por la incertidumbre y el temor al contagio y a la muerte.

Una característica más que otras patologías no presentan ha sido la puesta en práctica del confinamiento domiciliario como medida preventiva durante la pandemia ${ }^{1}$. Esta acción ha modificado hábitos y costumbres y ocurre de manera simultánea con otras crisis emocionales e instrumentales de la vida diaria. Al principio favoreció la unión solidaria en los hogares y la convivencia en medio de cierta armonía, tal vez con la idea de que su duración sería breve; sin embargo, un par de semanas bastaron para que se presentaran el desencanto, la frustración, el enojo y el tedio. Con el paso de los días, aumentaron la incertidumbre, el hastío y se agregaron el mal aprovechamiento del tiempo, el sedentarismo, la disfunción familiar y la importante carga de estrés que conllevan la convivencia prolongada, la pobreza súbita, la ansiedad y la depresión. El mayor contacto entre las personas, delimitado a los confines físicos de las viviendas, ha aumentado el riesgo de violencia doméstica, sobre todo en familias habituadas al conflicto o en las que las agresiones interpersonales son frecuentes ${ }^{2}$. Adicciones, suicidios, trastornos del sueño, consumo de estupefacientes y psicotrópicos se han exacerbado desde el inicio de la propagación mundial de la COVID-19.

Los efectos de cualquier enfermedad aguda, crónica, transmisible, accidental o congénita, dependen de múltiples parámetros, entre ellos, los relacionados con el tipo de familia, fase del ciclo vital y funcionalidad familiar, nivel socioeconómico, escolaridad y ocupación de los padres, características de la vivienda y número de integrantes. Otros aspectos que considerar 
son la presencia de enfermedades crónicas, apego terapéutico, percepción de la enfermedad y sus consecuencias, disponibilidad de servicios médicos y acceso a las redes sociales de apoyo. Debido a esta multiplicidad de factores y a la gran diversidad de familias, las consecuencias suelen ser distintas y el grado de afectación, diferente. Los efectos de la actual pandemia no son la excepción, ya que también están en función de estos parámetros.

\section{LAS FAMILIAS EN MÉXICO}

Las familias en México son muy diferentes entre sí y reaccionan de distinta manera ante los problemas de salud y las crisis de la vida cotidiana. Según el Instituto Nacional de Estadística y Geografía, este año habrá en México 127 millones de habitantes en 35 millones de viviendas, de las cuales, cerca del 90\% conforman hogares familiares y el resto son unipersonales ${ }^{3-5}$. Los hogares familiares son nucleares con o sin hijos (64\%), extensos ascendentes o descendentes $(23 \%)$ y seminucleares maternos o pater$\operatorname{nos}(13 \%)^{3,6}$.

Las familias reconstituidas invariablemente comparten características con los demás tipos, de acuerdo con su conformación, en tanto que los hogares unipersonales son habitados por personas solas, ya sea por divorcio, separación, viudez o por soltería.

\section{IMPLICACIONES DE LA PANDEMIA EN EL CICLO DE VIDA FAMILIAR}

El ciclo vital de la familia consta de cuatro etapas, a saber: constitutiva, procreativa, dispersión y final ${ }^{6}$. La primera incluye la fase preliminar o noviazgo y la unión de la pareja por matrimonio o unión libre; la segunda comprende la expansión de la familia con el nacimiento del primer hijo y termina cuando nace o se adopta el último. La etapa de dispersión implica el desprendimiento del hogar de los hijos, con lo cual, la pareja queda nuevamente sola y pasa a las fases de independencia y disolución. Esta breve descripción corresponde a una familia nuclear con hijos, de manera que habrá tantas clasificaciones del ciclo vital como tipos de familias haya. En cualquier caso, estas características incidirán en los efectos de la pandemia y, al final, las modificaciones estructurales y funcionales familiares serán evidentes.

Las etapas del ciclo vital familiar tienen atributos propios y distintivos en los diferentes tipos de familias, y se relacionan directamente con los factores de riesgo presentes en cada una de ellas. En cada fase la dinámica familiar continúa su evolución, pero puede alterarse por los eventos críticos que pudieran surgir, y los conflictos exacerbarse ante las condiciones del confinamiento doméstico.

\section{LAS FAMILIAS, SUS EFECTOSY MODIFICACIONES POR LA PANDEMIA}

Las familias mayormente afectadas durante la crisis causada por la COVID-19 presentan baja escolaridad, pobreza y tienden a minimizar el problema epidemiológico. En sus integrantes se han encontrado problemas de salud como obesidad, diabetes mellitus, hipertensión arterial y otras enfermedades crónicas que constituyen importantes factores de riesgo.

Estadísticamente, las familias nucleares son las más afectadas por ser más frecuentes, por su precaria situación económica y por la frecuencia y magnitud de los conflictos causados por el confinamiento, sobre todo las que tienen hijos y se enfrentan a la gran diversidad de factores de riesgo en cada fase de su ciclo vital. Cuando aún no tienen hijos se encuentran en fase de unión de pareja; cuando los hijos nacen o se adoptan, pasan a las fases de expansión y dispersión, lo cual implica la presencia en el hogar de mujeres en edad reproductiva, niños pequeños y adolescentes. El personal médico debe detectar oportunamente durante la pandemia signos y síntomas infantiles producto de la disfunción familiar o de trastornos emocionales, como enuresis, bajo aprovechamiento escolar, agresividad o terrores 
nocturnos; en tanto que adicciones, violencia, embarazo y accidentes en la adolescencia son problemas que pueden presentarse o exacerbarse durante este periodo de crisis.

El fallecimiento del padre o la madre da lugar a la formación de una familia seminuclear contraída. Si el deceso ocurrió a causa del contagio del coronavirus, además de las implicaciones propias del proceso del duelo, del cuidado y las medidas de prevención correspondientes, la familia debe enfrentar los conflictos propios de la fase del ciclo vital familiar en que se encuentre, así como las dificultades económicas, domésticas y laborales y hacerse cargo de los hijos sin su pareja. Durante la pandemia, las familias de un solo padre pueden sufrir los efectos del desempleo, el problema del cuidado de hijos pequeños y los efectos del confinamiento, la frustración y el contacto constante y permanente de todos los miembros en el hogar. Estas situaciones pueden ser causa de maltrato infantil y de la exacerbación de otras manifestaciones psicológicas y sociales en todos los integrantes. Habitualmente, las familias seminucleares son encabezadas por mujeres solteras o que han enfrentado, aparte de la viudez, otras causas de contracción familiar como el divorcio o la separación, de manera que al evaluarlas se debe investigar el motivo de la contracción, tiempo de evolución y superación o no del evento crítico.

En estos tiempos es muy probable que una gran cantidad de familias nucleares y seminucleares pasen a ser extensas al conjuntarse las de origen con las de procreación, ya sea en el hogar de unos o en el de otros, con la finalidad de solventar dificultades económicas, compartir vivienda o favorecer el cuidado de niños y viejos. Son ascendentes cuando la familia de uno de los hijos va a vivir con sus padres, en tanto que en las descendentes estos se alojan con alguno de sus hijos. La conjunción de más de dos núcleos familiares incrementa significativamente la presencia de factores de riesgo, ya que cada uno presenta características y conflictos propios de la fase del ciclo vital en que se encuentren. La pobreza, el hacinamiento y la promiscuidad habitacional, frecuentes en este tipo de familias, pueden aumentar los conflictos relacionales y el consumo de alcohol ${ }^{7}$, y ocasionar violencia, maltrato y distanciamiento afectivo. Los adultos mayores que suelen formar parte de estas familias podrían generar o exacerbar gran parte de los problemas domésticos, así como el deficiente control de sus enfermedades crónicas y el mal apego terapéutico, que tienden a ser habituales en momentos críticos. Debido al actual fenómeno epidemiológico, es muy probable que paulatinamente se incremente el número de convivientes en los hogares extensos que ya existen.

Otro aspecto relevante lo representan quienes habitan en hogares unipersonales, obviamente, sin compañía. La soledad puede ser un factor de riesgo muy importante en estos días, sobre todo en adultos mayores o en personas que presentan enfermedades invalidantes, por lo que en esta crisis resultan de gran importancia las redes sociales de apoyo por la ayuda que pueden otorgar, tanto en aspectos económicos, como de asistencia y compañía.

Las familias reconstituidas comparten algunas características con las nucleares, aunque las diferencias son importantes; también con las extensas, si en la vivienda hay miembros de las familias de origen o de procreación. Se conforman por personas que han tenido uniones previas, hayan procreado hijos o no y que ahora pueden ser viudos, divorciados o separados si vivían en unión libre. Los problemas y conflictos pueden surgir entre los medios hermanos y hermanastros, así como por la edad, ocupación y el tipo de relación que lleven estos entre sí y con sus padres y padrastros. Los desacuerdos y las expectativas de la pareja, así como el grado de funcionalidad, cohesión y adaptabilidad en estas familias, son elementos importantes para el mantenimiento de la homeostasis en el hogar, la cual puede afectarse o verse favorecida por la presencia y resolución de ciertos eventos críticos, como el nacimiento de un hijo. Este hecho podría incrementar en la pareja el sentimiento de inclusión en beneficio de la relación parental 
y entre ellos, situación que, ante la crisis actual, podría mantener o mejorar la funcionalidad en estas familias reconstituidas.

La crisis epidemiológica causada por el coronavirus ha demostrado que la vida puede cambiar en cualquier momento. Ha representado un gran reto para las familias que se han adaptado para enfrentar situaciones desconocidas y complejas y desarrollar diversos grados de resiliencia. En las personas y en las familias, la resiliencia es la capacidad que les permite superar tragedias o situaciones traumáticas y se enfoca más en el proceso de cómo superar la crisis que en el resultado, que no necesariamente será siempre positivo. Un adecuado nivel de resiliencia favorece que haya menos situaciones negativas, sin embargo, después de un evento crítico no todas las personas recuperan totalmente sus características y circunstancias originales, porque invariablemente quedan huellas, cicatrices o recuerdos, como habrá de suceder después de que este fenómeno epidemiológico haya sido superado. La resiliencia no evitará situaciones como la pandemia, pero permitirá abordarla de una manera más rápida y efectiva y favorecerá el aprendizaje para aminorar las consecuencias negativas.

\section{EL MÉDICO FAMILIAR ANTE LA PANDEMIA}

La salud está influida por la vida familiar y las familias se afectan por las enfermedades de sus miembros ${ }^{8}$, que en general repercuten en todo el conjunto familiar ${ }^{9}$. La familia es la unidad básica de la enfermedad y la salud, por lo que, en la práctica médica, debe ser la unidad mínima de estudio ${ }^{10,11}$, por ser el ambiente más importante en el que ocurren y se resuelven los problemas de salud ${ }^{12}$. Esto confirma que el quehacer del médico familiar, lejos de concretarse exclusivamente en la atención del individuo enfermo, debe centrarse en los factores de riesgo de la familia, como el principal objetivo de su quehacer profesional.

Los múltiples problemas psicosociales que giran en torno a las familias por la presencia de la COVID-19 requieren un abordaje integral que incluya el trabajo conjunto con la familia y la intervención oportuna y eficaz del personal de salud. Las diferencias familiares ameritan intervenciones concretas y específicas, además de la atención por el contagio del coronavirus o el fallecimiento por esta causa. El modelo de atención centrado en la familia es un proceso que permite llevar a cabo de manera sistematizada el trabajo del médico familiar. Requiere en primera instancia, atender y resolver los problemas de salud del paciente y simultáneamente, por medio de un familiograma, identificar el tipo de familia, sus integrantes, fases del ciclo vital y los factores de riesgo que, una vez jerarquizados, deben ser eliminados o atenuados.

La naturaleza altamente transmisible del coronavirus ha afectado a la participación familiar en rituales y actividades post mortem, debido a las restricciones en viajes, funerales y entierros; así mismo, la mayor posibilidad de fallecimientos múltiples y lo súbito de los decesos ha dificultado el proceso de duelo ${ }^{13}$. La presencia de un familiar grave o fallecido durante la pandemia requiere un adecuado abordaje afectivo y comprensivo por parte del personal de salud, sobre todo por las limitaciones implementadas y el distanciamiento social que, aunque necesarios, complican este proceso. Ante esta situación, se debe recomendar a los miembros de la familia de la persona fallecida evitar el aislamiento, mantener la comunicación efectiva con grupos de apoyo, manejar el duelo anticipado, evitar en la medida de lo pertinente la hospitalización y las intervenciones intensivas innecesarias, y solicitar apoyo de manera oportuna.

El médico familiar y el equipo de salud deben orientar a la familia acerca de qué hacer si se presentan síntomas sugestivos de infección por coronavirus 2 del síndrome respiratorio agudo grave (SARS-CoV-2), cómo sería la atención médica domiciliaria o qué hacer si el cuadro clínico se agrava. Así mismo, evitar el contacto cercano con personas enfermas, quedarse en casa y si hubiera necesidad de salir, hacerlo con las debidas precauciones. 
A medida que los contagios disminuyan, recomendar la continuidad del uso constante y correcto de cubrebocas, el lavado frecuente de manos, efectuar las medidas preventivas al toser o estornudar, evitar aglomeraciones, mantener la distancia adecuada con otras personas y evitar tocarse la cara. También se debe fomentar el dormir lo suficiente, practicar ejercicio físico, manejar correctamente el estrés y mantener una hidratación y alimentación adecuadas. En cuanto a la incertidumbre y al temor, que van siendo parte de nuestra vida, es muy importante reconocerlos y entenderlos para hablar de ello con el paciente y su familia. La comprensión y el apoyo nos beneficia a nosotros mismos.

Estas deberán ser, a partir de ahora, algunas de las acciones que el personal de salud y las familias han de llevar a cabo, como parte de la modificación de hábitos y costumbres que favorecerán la rehabilitación, el mantenimiento de un estado de salud óptimo y la atenuación y prevención de los efectos que la pandemia por la COVID-19 ha causado.

\section{FINANCIAMIENTO}

El autor no recibió patrocinio para llevar a cabo este artículo.

\section{CONFLICTO DE INTERESES}

El autor declara no tener conflicto de intereses alguno.

\section{RESPONSABILIDADES ÉTICAS}

Protección de personas y animales. Los autores declaran que para esta investigación no se han realizado experimentos en seres humanos ni en animales.
Confidencialidad de los datos. Los autores declaran que han seguido los protocolos de su centro de trabajo sobre la publicación de datos de pacientes.

Derecho a la privacidad y consentimiento informado. Los autores declaran que en este artículo no aparecen datos de pacientes.

\section{BIBLIOGRAFÍA}

1. Nicola M, Alsafi Z, Sohrabi C, Kerwan A, Al-Jabir A, losifidis $C H$, et al. The socio-economic implications of the coronavirus pandemic (COVID-19): A review. Int J Surg. 2020;78:185-93.

2. Usher K, Bhullar N, Durkin J, Gyamfi N, Jackson D. Family violence and COVID-19: Increased vulnerability and reduced options for support. Int J Ment Health Nurs. 2020;29:549-52.

3. En México hay 34.1 millones de hogares; $28.5 \%$ con jefatura femenina: encuesta nacional de hogares 2017. Comunicado de prensa núm. 251/18 [Internet]. México: Instituto Nacional de Estadística y Geografía; 28 de mayo del 2018. Disponible en: https://www.inegi.org.mx/contenidos/saladeprensa/boletines/2018/EstSociodemo/enh2018 05.pdf

4. Pasquali M. México: población total desde 2014 hasta 2024 [Internet]. Statista [fecha de acceso: 10/09/2020]. Disponible en: https://es.statista.com/estadisticas/635250/poblacion-total-de-mexico-en-2020/

5. La composición de las familias y hogares mexicanos se ha transformado en décadas recientes como resultado de cambios demográficos y sociales [Internet]. Gobierno de México: Consejo Nacional de Población; 15 de mayo de 2020. Disponible: https://www.gob.mx/conapo/articulos/la-composicion-de-las-familias-y-hogares-mexicanos-se-ha-transformado-en-las-recientes-decadas-como-resultado-de-cambios-demograficos?idiom=es

6. Huerta-González JL. Medicina familiar. La familia en el proceso salud-enfermedad. México: Alfil; 2005.

7. Chodkiewicz J, Talarowska, Miniszewska J, Nawrocka, N, Bilinski P. Alcohol consumption reported during the COVID-19 pandemic: The initial stage. Int J Environ Res Public Health. 2020;17:4677.

8. McWhinney IR. Family medicine un perspective. N Engl J Med. 1975;293:676-82.

9. Somolinos PJ. La medicina y la mujer en la conquista de México. Gac Med Mex. 1984;120:213-28.

10. Ackerman NW. Diagnóstico y tratamiento de las relaciones familiares: psicodinamismos de la vida familiar. 7. ${ }^{\mathrm{a}}$ ed. Buenos Aires, Argentina: Ediciones Hormé; 1982.

11. Sauceda GJ, Foncerrada MM. La Exploración de la familia. Aspectos médicos, psicológicos y sociales. Rev Med IMSS. 1981;19:155-63.

12. Horwitz CN, Florenzano UR, Ringeling PI. Familia y salud familiar. Un enfoque para la atención primaria. Bol Of Sanit Panam. 1985;98(2):144-55.

13. Araujo Hernández M, García Navarro S, García-Navarro EB. Abordaje del duelo y de la muerte en familiares de pacientes con COVID-19: revisión narrativa. Enferm Clin [Internet]. En prensa, 19 de mayo de 2020. Disponible en: https://www.sciencedirect.com/science/article/pii/S1130862120303089?via\%3Dihub 